\title{
Voglibose for prevention of type 2 diabetes mellitus
}

\author{
André J Scheen ${ }^{1}$ \\ Division of Diabetes, Nutrition and Metabolic Disorders, \\ Department of Medicine, CHU Sart Tilman, University of Liège, \\ B-4000 Liège, Belgium
}

Lifestyle interventions are effective for the prevention of type 2 diabetes in different populations with impaired glucose tolerance in the USA, ${ }^{1}$ north Europe ${ }^{2}$ China, ${ }^{3}$ and Japan. ${ }^{4}$ Not only were the results impressive at the end of the intensive intervention trials, but long-term follow-up studies also confirmed the effect of lifestyle interventions for the prevention of diabetes in individuals at high risk of developing the disease. ${ }^{2,3}$ Various drugs are efficacious in the prevention of type 2 diabetes in individuals with impaired glucose tolerance (figure). Glucose-lowering drugs preventedthe development of type 2 diabetes in placebo-controlled clinical trials. ${ }^{1,3}$ Metformin reduced the relative risk of new-onset diabetes at the end of the US Diabetes Prevention Programme by $31 \%{ }^{1}$ and a $26 \%$ reduction was seen inthe Indian Diabetes Prevention Programme. ${ }^{8}$ Treatment with thiazolidinediones resulted in a greater risk reduction $\left(60 \%\right.$ with rosiglitazone in DREAM, ${ }^{11}$ and $81 \%$ with pioglitazone in ACT-NOW $\left.{ }^{12-14}\right)$. However, because both metformin and glitazones positively affect the pathophysiology of type 2 diabetes, ${ }^{15}$ and because the main reported results were analysed without any washout period, the distinction between a masking and atrue preventive effect is almost impossible. ${ }^{16}$

Another approach that would not directly affect insulin sensitivity or insulin secretion is to competitively inhibit intestinal $\alpha$-glucosidase enzymes. ${ }^{17}$ In the STOP-NIDDM trial, ${ }^{6}$ done in Canada and various European countries, a $25 \%$ reduction was reported in the number of patients with new-onset diabetes and impaired glucose tolerance who were given acarbose (100 mg three times a day) compared with those given placebo after a 3-year followup. ${ }^{6}$ Furthermore, acarbose significantly increased reversion of impaired glucose tolerance to normal. However, at the end of the study, treatment with placebo for 3 months was associated with an increase in conversion of impaired glucose tolerance to diabetes. Because acarbose does not interfere with the intestinal absorption of the monosaccharide glucose that is ingested during the oral glucose tolerance test, a masking effect seems unlikely compared with previous discussions about metformin or glitazones. ${ }^{16}$

$\alpha$-glucosidase inhibitors seem to exert a good glucose-lowering effect (with an acceptable gastrointestinal tolerance) in Asian people, presumably because of specific food habits. ${ }^{18}$ Therefore the results of the trial by Kawamori and colleagues in The Lancet today, in which the effect of voglibose (another $\alpha$-glucosidase inhibitor) was assessed in Japanese patients with impaired glucose tolerance, raise much interest. ${ }^{5}$ Analysis after almost 50 weeks of treatment showed that voglibose $(02 \mathrm{mg}$ three times a day) reduced the risk of patients with impaired glucose tolerance developing type 2 diabetes by $40.5 \%$ and increased the proportion who achieved normoglycaemia by $53.9 \%$ compared with those given placebo. The underlying mechanism of action is not known. Unlike metformin and glitazones, a direct effect on insulin sensitivity or insulin secretion is unlikely. However, as with acarbose in STOP-NIDDM, ${ }^{6}$ voglibose might reduce diurnal insulin secretion through improvement of postprandial hyperglycaemia, thereby reducing the stress on overworked $\beta$ cells (figure). ${ }^{15,19}$

Is voglibose more effective than intensive lifestyle changes in these Japanese patients, ${ }^{4}$ in view of the fact that drug treatment is more expensive and might be associated with adverse events (mostly gastrointestinal symptoms with $\alpha$-glucosidase inhibitors, although in today's study reported as mild to moderate)? This question cannot be answered because the objective of today's study was to assess whether the addition of voglibose to a standard diet and regular exercise could improve clinical outcomes compared with diet and exercise alone. Indirect comparison of the findings of the two Japanese trials, in which intensive lifestyle intervention (risk reduction $67 \cdot 4 \%)^{4}$ or voglibose plus lifestyle instructions $(40 \cdot 5 \%$, today's study) on the new onset of diabetes in individuals with impaired glucose tolerance was assessed, might be criticised. However, lifestyle changes remain a cornerstone in all strategies for prevention of type 2 diabetes. ${ }^{1-4}$ Today's Japanese study showed that, if best efforts to educate individuals do not work, treatment with voglibose could be an important way forward. Could

\footnotetext{
${ }^{1}$ I am a consultant for Sanofi-Aventis, AstraZeneca, GlaxoSmithKline, and have received lecture fees from Sanofi-Aventis.
} 
the results obtained with voglibose in Japanese patients be extrapolated to other populations, in view of the well known good efficacy and safety profile of $\alpha$-glucosidase inhibitors in Asians? ${ }^{18}$ The answer might be yes because the reported results agree with those of the STOP-NIDDM trial with acarbose in Canadians and Europeans. ${ }^{6}$ With the positive results obtained with metformin ${ }^{1,8,12}$ and $\alpha$-glucosidase inhibitors (today's study and references 8 and 19) on the one hand, and the rather high residual risk of developing type 2 diabetes with monotherapy on the other, treatment with a combination of the two types of drugs might be an option. Such a combined metformin-acarbose approach is being tested in a trial in progress in Canada (CANOE). ${ }^{20}$

Figure: Prevention of type 2 diabetes in high-risk individuals with impaired glucose tolerance Drug effects, possible mechanisms, and clinical trials. Data within parentheses are reductions in relative risk of new-onset diabetes in active versus placebo arm.

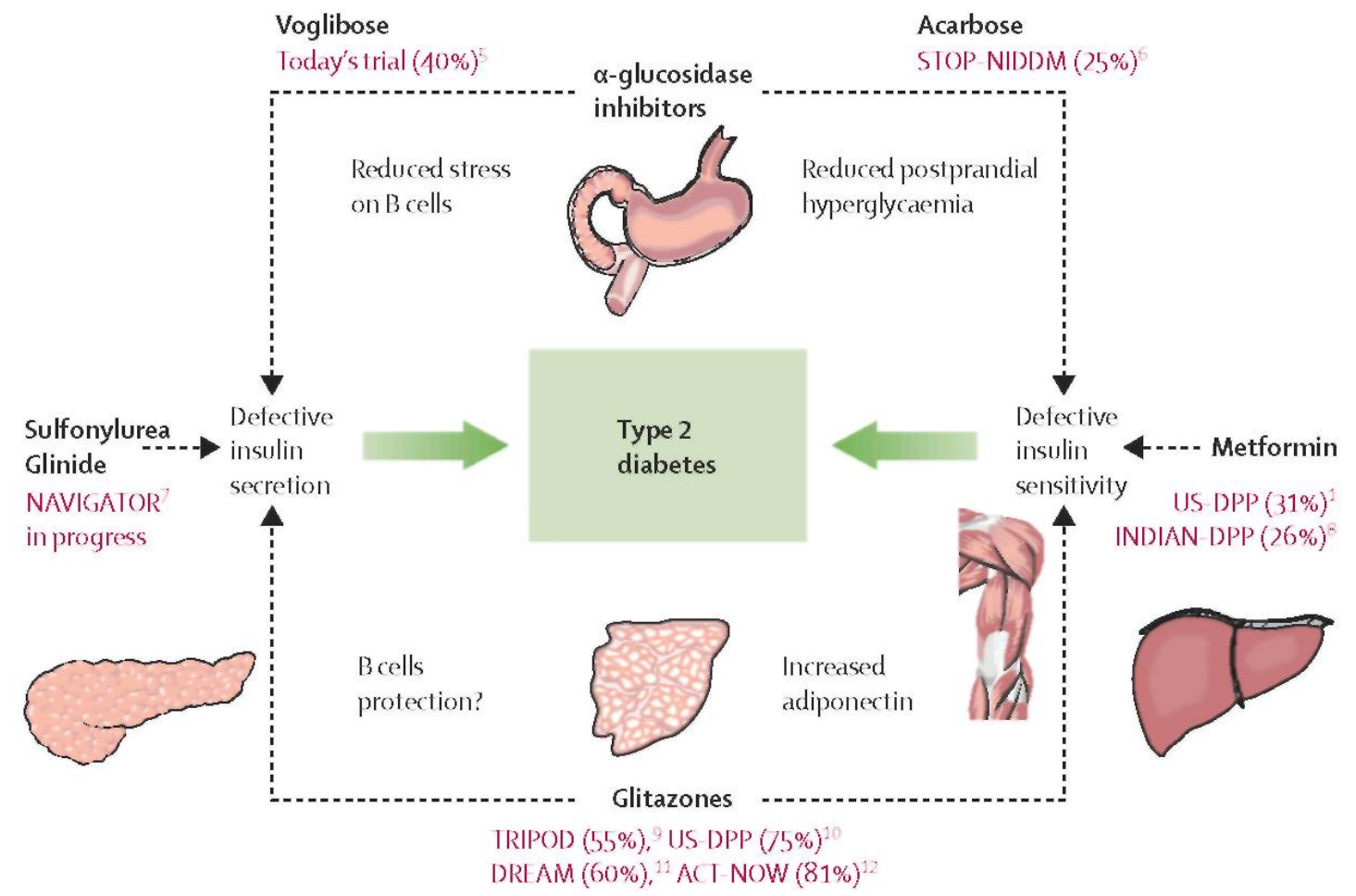

Why the independent data-monitoring committee decided to end the voglibose study early is not known. One might be able to understand such a decision when the primary endpoint included major adverse cardiovascular events or mortality, but not when (as here) the endpoint is transition from impaired glucose tolerance to diabetes. This decision ledtoa much shorter follow-up $(<1$ year) in this study than in other trials. Additionally, early stopping of randomised trials for no overt efficacy can be problematic and can lead to an overestimation of the difference between the treatment arms. ${ }^{21}$ Whether these preventive approaches can slow the development of vascular complications of diabetes and ultimately reduce diabetes-related mortality remains an open question.

\section{References}

1 Diabetes Prevention Program Research Group. Reduction in the incidence of type 2 diabetes with lifestyle intervention or metformin. $N$ Engl J Med 2002; 346: 393-403.

2 Lindström J, llanne-Parikka P, Peltonen M, et al, on behalf of the Finnish Diabetes Prevention Study Group. Sustained reduction in the incidence of type 2 diabetes by lifestyle intervention: follow-up of the Finnish Diabetes Prevention Study. Lancet 2006; 368:1673-79. 
4 Kosaka K, Noda M, KuzuyaT Prevention of type 2 diabetes by lifestyle intervention: a Japanese trial in IGT males. Diabetes Res Clin Pract 2005; 67:152-62.

5 Kawamori R, Tajima N, IwamotoY, et al, on behalf of the Voglibose Ph-3 Study Group. Voglibose for prevention of type 2 diabetes mellitus: a randomised, double-blind trial in Japanese individuals with impaired glucose tolerance. Lancet 2009; published online April 22. D0I:10.1016/ S0140-6736(09)60222-1.

6 ChiassonJ-L, Josse RG, Gomis R, Hanefeld M, Karasik A, Laakso M, for the STOP-NIDDMTrial Research Group. Acarbose for prevention of type 2 diabetes mellitus: the STOP-NIDDM randomised trial. Lancet 2002; 359: 2072-77.

7 Califf RM, Boolell M, Haffner SM, et al, for the NAVIGATOR Study Group. Prevention of diabetes and cardiovascular disease in patients with impaired glucose tolerance: rationale and design of the Nateglinide And Valsartan in Impaired Glucose Tolerance Outcomes Research (NAVIGATOR) Trial. Am Heart J 2008; 156: 623-32.

8 Ramachandran A, Snehalatha C, Mary S, Mukesh B, Bhaskar AD, Vijay V, and Indian Diabetes Prevention Programme (IDPP). The Indian Diabetes Prevention Programme shows that lifestyle modification and metformin prevent type 2 diabetes in Asian Indian subjects with impaired glucose tolerance (IDPP-1). Diabetologia 2006; 49: 289-97

9 Buchanan TA, Xiang AH, Peters RK, et al. Preservation of pancreatic beta-cell function and prevention of type 2 diabetes by pharmacological treatment of insulin resistance in high-risk Hispanic women. Diabetes 2002;51:2796-803.

10 TroglitazoneThe Diabetes Prevention Program Research Group. Prevention of type 2 diabetes with troglitazone in the Diabetes Prevention Program. Diabetes 2005; 54:1150-56.

11 The DREAM (Diabetes Reduction Assessment with ramipril and rosiglitazone Medication) Trials Investigators. Effect of rosiglitazoneon the frequency of diabetes in patients with impaired glucose tolerance or impaired fasting glucose: a randomised controlled trial. Lancet 2006; 368:1096-105.

12 Anon. Actos Now for Prevention of Diabetes (ACT-NOW). Feb 16, 2006. http://clinicaltrials.gov/ct2/show/NCTOO220961 (accessed March 10, 2009).

13 Scheen AJ. Pharmacological prevention of type 2 diabetes. In: EkoeJ-M, Rewers M, Williams R, Zimmet P, eds. The epidemiology of diabetes mellitus, 1st edn. London: Wiley-Blackwell, 2008: 449-74.

14 Beals JK. Pioglitazone reduces conversion from impaired glucose tolerance to type 2 diabetes, http://www.medscape.com/viewarticle/575860 (accessed March 30, 2009).

15 Stumvoll M, Goldstein BJ, van Haeften TW. Type 2 diabetes: principles of pathogenesis and therapy. Lancet 2005; 365:1333-46.

16 Scheen AJ. Antidiabetic agents in patients with mild dysglycaemia: prevention or early treatment of type 2 diabetes? Diabetes Metab $2007 ; 33: 3-12$

17 Scheen AJ. Is there a role for $\alpha$-glucosidase inhibitors in the prevention of type 2 diabetes? Drugs 2003; 63: 933-51.

18 Chan JC, Chan KW, Ho LL, et al, for the Asian Acarbose Study Group. An Asian multicenter clinical trial to assess the efficacy and tolerability of acarbose compared with placebo in type 2 diabetic patients previously treated with diet. Diabetes Care 1998; 21: 1058-61.

19 Nijpels G, Boorsma W, DekkerJM, et al. A study of the effects of acarbose on glucose metabolism in patients predisposed to developing diabetes: the Dutch acarbose intervention study in persons with impaired glucose tolerance (DAISI). Diabetes Metab Res Rev 2008; 24: 6II-I6.

20 Zinman B, Harris SB, Gerstein HC, et al. Preventing type 2 diabetes using combination therapy: design and methods of the Canadian Normoglycaemia Outcomes Evaluation (CANOE) trial. Diabetes Obes Metab 2006; 8: 531-37

21 Bassler D, Montori VM, Briel M, et al. Early stopping of randomized trials for overt efficacy is problematic. J Clin Epidemiol 2008; 61: 241-46. 